\title{
Aprendizaje Electrónico Afectivo: un modelo Innovador para Desarrollar una Acción Tutorial Virtual de Naturaleza Inclusiva
}

\author{
Alba M. Hernández-Sánchez y José A. Ortega \\ Grupo de Investigación Tecnología Educativa e Investigación Social (TEIS), Departamento de Didáctica y \\ Organización Escolar. Facultad de Ciencias de la Educación. Universidad de Granada. Campus \\ Universitario de Cartuja, s/n, 18071. Granada - España (e-mail: albamaria@ugr.es, jaorte@ugr.es)
}

Recibido Jul. 21, 2014; Aceptado Sep. 25, 2014; Versión final recibida Nov. 17, 2014

\begin{abstract}
Resumen
Este trabajo propone líneas de acción tutorial para una educación a distancia inclusiva en entornos virtuales. Se describe una experiencia formativa en línea en la que ha participado un grupo de alumnado y de tutores formado por personas sordas y oyentes en una atmósfera de aprendizaje electrónico afectivo (affective eLearning) Los resultados han surgido de la triangulación de información obtenida de las tutoras intervinientes mediante entrevistas y narraciones autobiográficas. Se pone énfasis en una tutoría afectiva de calidad a través del manejo del tiempo y el espacio de forma flexible. La metodología de trabajo está orientada a la generación de emociones positivas, al desarrollo de una evaluación comprehensiva y humanizadora y a la materialización de estrategias que favorezcan el bienestar y la empatía con el grupo.
\end{abstract}

Palabras clave. aprendizaje electrónico afectivo, psicología positiva, educación inclusiva, tutoría virtual, discapacidad auditiva

\section{Affective e-Learning: an Innovative Model for Developing a Mentoring Virtual Action in an Inclusive Context}

\begin{abstract}
This work proposes lines of mentoring action to be used in a virtual distance education context. An on-line formative experience with participation of students and teachers with hearing and non-hearing capacity in an affective e-Learning atmosphere is described. The outcomes have come from the triangulation applied to the information gathered by the participating teachers through interviews and autobiographic narrations. Emphasis is put on an affective mentoring managing time and space in a flexible form. The methodology is aimed to the creation of positive emotions, to the development of a comprehensive and humanizing evaluation and to the materialization of strategies that encourage well-being and empathy among the group.
\end{abstract}

Keywords: affective e-learning, positive psychology, inclusive education, virtual mentoring, hearing disability 


\section{INTRODUCCIÓN}

El análisis de los flujos emocionales en la enseñanza a distancia en entornos virtuales ha sido escasamente estudiado hasta la fecha, si bien las propuestas de Shen et al., (2009) constituyen un precedente valioso en este ámbito de conocimiento. El modelo de eLerning afectivo ha ido surgiendo tras un decenio de investigaciones e innovaciones realizadas en el seno del Grupo Internacional de Investigación Tecnología Educativa e Investigación Social (TEIS), coordinado por la Universidad de Granada. En el mismo, los desarrollos emocionales y afectivos se consideran claves (O'Regan, 2003) como catalizadores de los proceso de aprendizaje, para desde la mejora de la atención, la memoria y la toma flexible de decisiones, resolver problemas y mejorar la eficiencia y el rigor para decidir ante situaciones concretas (Shen, Wang y Shen, 2009).

Los enfoques propios de la Psicología Positiva que fundamenta el modelo eLerning afectivo permite que las conductas afectivas de los diferentes actores produzcan un estado generalizado de bienestar sostenido por la consecución de un permanente estado de flujo. Entendiendo que el flujo es "un estado subjetivo que las personas experimentan cuando están completamente involucradas en algo hasta el extremo de olvidarse del tiempo, la fatiga y de todo lo demás, excepto de la actividad en sí misma" (Csikszentmihalyi, 2009, En este loable propósito colectivo juega un papel destacadísimo el ejercicio de una acción tutorial generadora de emociones y sentimientos positivos que motiven al alumnado a resolver las dificultades y a superar los momentos de desánimo, incidiendo en el cumplimiento de la función adaptativa que producen los estados de ánimo y las emociones positivas (Vázquez, 2009); reforzando así su capacidad de resiliencia.

Por tal motivo, en el ejercicio del desempeño tutorial afectivo, éste es uno de los focos principales de atención de esta investigación por considerarse como factor "clave para el éxito de un programa formativo" (Arranz, Aguado y Lucía, 2008, Consiguientemente, el desarrollo de la tutoría virtual se entiende como un elemento crucial para adaptar el aprendizaje a las peculiaridades del contexto y de los participantes, al actuar el tutor como mediador y guía entre el alumnado y los contenidos de aprendizaje (Seoane y García, 2007). La información no se incorpora de forma automática a nuestro conocimiento sino que se precisa de la asistencia del profesor o experto (Njenga y Fourie, 2010) que medie en el proceso de aprendizaje. Mediación que se valora en la percepción de satisfacción del alumnado cuando: se responde rápidamente, su estilo de enseñanza es suficientemente bueno, sus explicaciones son claras, tiene bajo control el dominio de la tecnología (Ozkan y Koseler, 2009) y procede a la aplicación de test de diagnóstico previos que permitan "diseñar recursos y materiales publicados en el aula virtual utilizando las herramientas de aprendizaje ajustadas a los estilos de aprendizaje de los estudiantes" (Puello et al., 2014).

En las dos últimas décadas, han sido diversos los estudios que han perfilado las funciones de la tutoría virtual convencional. Así, Cabero (2004) y Llorente (2006) señalan las funciones o roles del tutor virtual relacionándolos con los ámbitos académico o pedagógico, social, organizativo o de dirección, orientador y técnico. Igualmente, García Aretio (2007) y Escalante (2012) enfatizan un conjunto de cualidades que propician el óptimo desarrollo de la labor tutorial a distancia, centradas en el mantenimiento de la cordialidad, la flexibilidad, la aceptación, la tolerancia, el respecto, la autenticidad, la honradez, la empatía, la capacidad de estar y de leer al otro, así como la capacidad de autocrítica. Tales cualidades son de suma importancia para facilitar los procesos de guía del aprendizaje identificando las áreas de acuerdo y desacuerdo, para tratar de llegar a consensos, reforzando las contribuciones de los estudiantes, estableciendo un clima adecuado, dibujando las situaciones problemáticas y evaluando la eficacia del proceso (Garrison y Anderson, 2005).

En el modelo eLerning afectivo, los procesos educativos nunca se conciben desde inercias mecánicas e irreflexivas, sino que se orientan desde el diseño dialogado y colaborativo de "estrategias educativas que impliquen el desarrollo de habilidades tanto cognitivas y meta-cognitivas, como prácticas éticas y prácticas desarrolladoras de inteligencia emocional y afectiva" (Cardona, 2008). Se pone el énfasis en el dominio afectivo que, desde las teorías constructivistas, entiende que el estudio de los aspectos afectivos y emocionales son la clave para explorar cuando uno trata de entender e interpretar la experiencia del alumnado (Zaharias y Poylymenakou, 2009), permitiendo que las condiciones de flujo se experimenten como el: "establecimiento de un sistema claro de metas, aportación de información retroactiva inmediata y establecimiento de un balance entre los retos percibidos y las habilidades percibidas" (Csikszentmihalyi, 2009).

Planteamientos que se refuerzan desde el ejercicio de una cultura inclusiva capaz de garantizar la participación de todo el alumnado propenso a la exclusión, desde la mejora de los entornos de aprendizaje, fomentando la participación de la comunidad y promoviendo el entendimiento de la diversidad como recurso positivo para el aprendizaje (Booth y Ainscow, 2000). Por ello, pensamos con Klinguer, Mejía y Posada, (2011) que las prácticas educativas inclusivas tienen que estar íntimamente ligadas a la responsabilidad del 
equipo docente a través de procesos de sensibilización y de desarrollo de competencias afectivas. Entendiendo que uno de los sustentos que da sentido a la diversidad como eje de la educación inclusiva es la defensa de la "teoría constructivista por los docentes donde el aprendizaje ha de ser una auténtica construcción que realiza el alumno mediada socialmente por el profesor, sus compañeros, sus conocimientos precios y su contexto" (Echeita et al., 2013). Si se realiza una extrapolación de la propuesta integradora que propone Tarragona (2013) entre las terapias constructivistas y la psicología positiva al ámbito educativo que estudia la discapacidad y la inclusión, se podría confirmar la importancia de esta relación que promueve un trabajo centrado en soluciones y no en la patología, considerando a la persona como experta de su propia vida y, por ello, protagonista de su propio proceso de aprendizaje.

Las posibilidades tecnológico-didácticas que ofrece la enseñanza a distancia en entornos virtuales para poner en práctica sistemas de comunicación hipermedia asíncronos y síncronos mediante el uso del texto, la imagen estática, el audio y el vídeo, incide en la generación de espacios donde la comunicación puede llegar a ser expresiva, cercana y cálida (Bautista et al., 2006). Nuestras experiencias de tutoría afectiva han puesto de manifiesto que tal calidez y cercanía han reforzado los estados emocionales positivos del alumnado aumentando su disfrute en el proceso de aprendizaje, contrastando que "la cantidad de disfrute que los profesores y profesoras obtienen de la docencia se relaciona con la cantidad de atención que los estudiantes muestran" (Rubio y García, 2010). La inmersión del alumnado en el denominado estado de flujo produce como resultado la "unión de la acción y la conciencia, un sentido de control y el sentido alterado del tiempo" (Csikszentmihalyi, 2009), lo que confirma que la experiencia de aprendizaje produce un bienestar sostenido que favorece el proceso de aprendizaje.

La investigación que se presenta se desarrolló en el trascurrir de la II Edición del Curso de Especialización en Lengua de Signos Española y su Interpretación aplicada a la enseñanza presencial y virtual (nivel A), a través de la Escuela Internacional de Posgrado de la Universidad de Granada. Tal acción formativa se llevó a cabo en el curso académico 2011/2012, en el entorno de aprendizaje denominado MUNDOSIGNO, un campus virtual accesible para personas sordas propiedad de la Fundación Andaluza de Accesibilidad y Personas Sordas, institución que colaboró en el desarrollo del proyecto.

\section{MÉTODOLOGÍA}

El propósito central de la investigación fue comprender y describir los modos de actuar del equipo tutorial formado por personas sordas y oyentes que participaron en la citada experiencia formativa a distancia en los momentos iniciales de surgimiento del modelo de e-Learning afectivo, como atmósfera envolvente del desarrollo de la pretendida práctica educativa inclusiva. Para conseguir tal finalidad se aplicaron dos estrategias de investigación que posibilitan la recopilación de información de forma profunda a las tutoras especialistas en la enseñanza de la lengua de signos española y con experiencia docente en entornos virtuales de aprendizaje. Se llevaron a cabo dos entrevistas semi-estructuradas a una de las tutoras sordas y a la tutora oyente, además de recogerse una narración autobiográfica de la otra tutora sorda.

Para llevar a cabo la recogida de información se diseñó una entrevista semi-estructurada guiada basada en un diseño que permite la profundización en la comprensión de la temática (McMillan y Schumacher, 2005). La entrevista está formada por un conjunto de preguntas abiertas que indagan sobre la actuación tutorial desde la práctica "humanizadora" y afectiva. Por su parte, la narración autobiográfico-narrativa ha permitido obtener el relato de una informante clave en un "contexto determinado y desde la interpretación de sí mismo" (Mallimaci y Giménez, 2006). Se ha puesto en práctica pues, "un intercambio dinámico entre la teoría, los conceptos y los datos con retroinformación y modificaciones constantes de la teoría y de los conceptos, basándose en los datos obtenidos" que caracteriza el método cualitativo (Cook y Reichardt, 2005), para facilitar la comprensión y el descubrimiento del objetivo delimitado. Con la intención de reforzar la validez de la investigación se ha realizado una triangulación de las informaciones obtenidas para aumentar la credibilidad de los resultados, de modo que se minimice lo que se ha denominado desde la psicología como "error de estímulo" (Stake, 2006). El análisis detallado de las informaciones obtenidas ha permitido obtener interesantes hallazgos que se dan a conocer en este trabajo, con el fin de incentivar la reflexión del colectivo de profesionales e investigadores comprometidos en la promoción y desarrollo de experiencias a distancia en entornos virtuales inclusivas.

\section{RESULTADOS}

El acercamiento al modelo de tutoría afectiva en entornos virtuales inclusivos se ha estructurado en varios apartados motivado por las propuestas de Hernández-Sánchez y García (2013) y Ortega et al., (2014): Tutoría virtual y organización de los tiempos y el espacio, la metodología de la acción tutorial, la evaluación en la acción formativa, dominio de los lenguajes y de la gestualidad y la expresividad creativa y convergencia de los roles del tutor afectivo 


\section{Tutoría virtual y organización de los tiempos y el espacio}

Respecto a los cuestiones relacionadas con los espacios, se concluye que el/la tutor/a virtual propiciará la creación de espacios de trabajo colaborativo relajantes, felices, motivadores, plenos de serenidad y de templanza. Actualmente, el aprendizaje ubicuo posibilita la entrada a los entornos virtuales desde diferentes lugares y dispositivos habilitados con conexión a Internet; razón por la que es crucial incentivar no solo la generación de entornos accesibles a nivel técnico, sino la motivación del alumnado por permanecer en el entorno virtual de aprendizaje de forma remota.

De igual modo, la organización temporal de la acción formativa ha de basarse en el ejercicio de la flexibilidad, la disponibilidad y la adecuación a los diferentes ritmos de aprendizaje del alumnado. Por lo que el ejercicio de la tutoría virtual ajustará en lo posible los horarios de atención sincrónica (videoconferencia) y asincrónica (foro y vídeoforo) a las disponibilidades del alumnado. La realización de una evaluación diagnóstica previa y el seguimiento continuo, favorecerá el establecimiento de acuerdos que permitan la conciliación de los horarios de atención a las necesidades del grupo de forma dialogada y a ser posible consensuada. Para ello, es importante planear los tiempos desde una actitud flexible y adaptativa, para que el equipo tutorial pueda ayudar a su alumnado a organizarse eficientemente, proponiéndoles las adaptaciones curriculares y organizativas oportunas que eviten o disminuyan la ansiedad ante situaciones de retraso o de dificultades sobrevenidas.

\section{La metodología de la acción tutorial}

La metodología de trabajo propuesta ha de estar orientada al desarrollo de emociones positivas que favorezcan el estado de flujo necesario para generar un estado de bienestar que favorezca el desarrollo cognitivo del alumnado, fomentando las relaciones interpersonales afectivas a través del uso de las diferentes estrategias de comunicación (especialmente de la videoconferencia y de los encuentros presenciales cuando son posibles -blended learning-), y ayudando a dirigir sus propias emociones y las de sus compañeros (conciencia emocional), canalizándolas hacia el aumento de la auto y la inter motivación.

Subrayamos la importancia de la tutoría virtual en la promoción del desarrollo cognitivo del alumnado, ayudándolo a recordar experiencias y aprendizajes previos que estimulen el establecimiento de conexiones y andamiajes con los nuevos aprendizajes individuales y colaborativos. En este contexto, han de fomentarse las estrategias de reorganización creativa de emociones e ideas, actuando de guía en los procesos de cumplimiento de satisfacción de las expectativas puestas en el programa formativa. Siendo con ello faro y asidero recurrente en los procesos de maduración autónoma y grupal.

La presencia en los materiales educativos en línea (diseño tecnológico-didáctico) de una sabia combinación de estrategias didácticas transmisivas, interactivas y colaborativas desarrolladas en un ambiente de emociones positivas, ha de permitir al equipo tutorial fomentar un sano optimismo en las relaciones interpersonales del grupo, reforzando dinámicas para mejorar competencias socioemocionales generadoras de empatía. Tal flujo de optimismo se conseguirá desde la inter-superación de ciertos prejuicios y dosis de timidez en el establecimiento de intercambios comunicativos asíncronos y síncronos (miedo a grabarse con una cámara, a expresar emociones, a realizar tareas ante un grupo, etc). La cuidada planificación previa de las sesiones grupales (teniendo en cuenta objetivos emocionales e intelectuales) es un aspecto clave para promover un espacio de confianza y seguridad donde los miembros de la ciber-comunidad de aprendizaje puedan expresarse con libertad y respeto, cuidando la equidad en el uso de los turnos de palabra para evitar que quienes son más extrovertidos inhiban a los más introvertidos.

Consiguientemente, el equipo tutorial ha de actuar como ente motivador de aprendizajes felices incentivando la participación y bienestar colectivo desde la automotivación intelectual y afectiva, la valoración de la satisfacción producida por la consecución y el reconocimiento de tareas exitosas, la constante retroalimentación con mensajes de ánimo y refuerzo y la emisión de comentarios productivos de mejora. El equipo tutorial afectivo ayudará pues a impulsar la conciencia emocional del grupo, incentivando la autoconciencia, la conciencia social y las estrategias de evitación de emociones negativas y de regulación de las emociones positivas, ayudando a mejorar los autoconceptos, permitiendo que se hagan públicas las valoraciones positivas de los trabajos y de los comportamientos colectivos.

Igualmente, el equipo tutorial ha de impulsar sinergias positivas entre las ideas, las emociones y las acciones aprovechando las sesiones individuales y grupales de videoconferencia y presenciales en su caso, auspiciando catarsis colectivas que favorezca la vibración emocional e intelectual generadora de interés, compromiso, satisfacción y esperanza, mediante el poder seductor de la imagen, la relajación y las dinámicas lúdicas y humorísticas. La acción tutorial ha de estar acompañada de una especial sensibilidad, cuidado y atención, en coordinación con el equipo directivo y coordinador, en la detección de indicios de 
problemáticas personales delicadas y de situaciones pre-conflictivas en el la ciber-comunidad, actuando de forma cercana, amable, inteligente y manifestando disponibilidad para la atención afectiva y cultivo de la resiliencia resolutiva.

\section{La evaluación en la acción formativa}

Desde la acción tutorial afectiva, se ha de relativizar los procesos de evaluación, desdramatizándolos y humanizándolos, haciendo que el impacto emocional de la evaluación se convierta en un proceso que incida en la mejora individual y grupal a través de procedimientos transparentes y democráticos. Se precisa de una atmósfera de confianza y transparencia, en la que el consenso de las pautas y el conocimiento de criterios e instrumentos de evaluación surja de la inter-construcción consensuada con el alumnado. Ello favorecerá la inter-evaluación desde la realización del trabajo colaborativo y la autoevaluación de los actores implicados. Siendo igualmente normalizado por parte del equipo tutorial el hecho de ser evaluado por su propio alumnado de forma periódica (en cada módulo y al final del plan formativo) ayudando con ello a su mejora profesional.

Tal como hemos indicado anteriormente, el equipo tutorial evaluará las experiencias, emociones y conocimientos previos del alumnado para adaptar los materiales formativos, las actividades y las estrategias metodológicas al inicio y durante la acción formativa (reeditando puntualmente o de forma periódica). Nuestro modelo propone el establecimiento de un equilibrio dinámico entre la evaluación de los procesos emocionales e intelectuales desarrollados y en los resultados emocionales y de los aprendizajes, combinándose técnicas de recogida de datos cuantitativos y con las estrategias de acopio de información cualitativa. Entendida pues la evaluación como proceso de mejora capaz de incidir en la calidad y la satisfacción de la comunidad ciber-educativa, sus frutos y resultados se han de poner en común de forma continuada y transparente en un clima humanizador y afectivo, subrayando las valoraciones positivas de los procesos y frutos resultantes, así como de los comportamientos amables, solidarios y eficaces, para con ello reforzar el flujo de emociones positivas generadoras de optimismo y de felicidad colectiva saludable.

\section{Dominio de los lenguajes y de la gestualidad y la expresividad creativa}

El equipo tutorial ha de conocer y poner en práctica una serie de estrategias que favorezcan el bienestar y la empatía para con su grupo de alumnado en los intercambios comunicativos. Tanto en la comunicación a través de un soporte textual o de imágenes (asíncrono o síncrono) como mediante un soporte en vídeo (asíncrono o síncrono), es necesario que se incida en la transmisión positiva de los mensajes, incidiendo en el modo de escribir o de expresar verbal o gestualmente la información. La mayor parte de los aspectos negativos generados surgen de malentendidos al interpretar lo que se lee o ve, por esta razón, es de crucial importancia cuidar la emisión de mensajes en sintonía con un el modelo de eLearning afectivo.

De igual forma se incidirá en la guía y mediación en los mensajes generados por el grupo de alumnado, interviniendo si es necesario de forma asertiva en alguna disyuntiva producida en un espacio de trabajo colaborativo. Las discrepancias han de ser asumidas como un elemento más de la riqueza de pensamiento, siempre y cuando se respecten todos los puntos de vista y se incida en la reflexividad justificativa de las aportaciones. El equipo tutorial en estrecha colaboración con el equipo directivo y coordinador han de gestionar y prever posibles situaciones problema para incidir en una atención amable, cercana, confiada y abierta como modelo para el resto de participantes. Incidiendo en la generación de actos comunicativos creativos donde los medios tecnológicos se pongan al servicio e intensifiquen el despliegue de intercambios comunicativos motivantes.

Tanto la organización flexible y consensuada de los espacios y los tiempos, como el despliegue de una metodología docente afectiva donde la preocupación constante por el impulso de sinergias positivas, han de ser una práctica habitual. Tal flexibilidad ha de reforzarse por la incorporación de un modelo evaluativo orientado a la mejora continua y liberado de antiguas percepciones estresantes y limitantes para el alumnado. Igualmente se afianzará mediante la puesta en prácticas de estrategias comunicativas creativas que ahonden en la transmisión de mensajes asertivos. La generación de espacios comunicativos donde se pueda trabajar de forma colaborativa y cooperativa es un indicador de suma importancia en nuestro modelo que se afana por superar la creencia de que este tipo de enseñanza impide a muchos de ellos -alumnadoel establecer relaciones y situaciones de aprendizaje compartido o cooperativo (Suárez y Anaya, 2004), aspectos sine qua non para desarrollar una enseñanza inclusiva.

\section{Convergencia de los roles del tutor afectivo}

Con la intención de reforzar la presentación de los resultados, se explicita la gráfica referente al manejo de los roles que un tutor afectivo domina y pone en práctica en el ejercicio de su labor tutorial, evaluativa y 
organizativa. Se presenta un mapa de categorías que ha sido elaborado a través de la extracción de las mismas de manera inductiva (según iban emergiendo de las informaciones analizadas). En el citado estudio se puede observar la distribución de cada una de las categorías en función al valor que se les da como aspectos imprescindibles, prioritarios o importantes para el desarrollo de una acción tutorial afectiva:

Tabla 1. Distribución de las categorías inducidas de los resultados de investigación. Informante 1: Tutora oyente (entrevista). Informante 2: tutora sorda (entrevista). Tutora 3: tutora sorda (historia de vida).

\begin{tabular}{|c|c|c|c|}
\hline & $\begin{array}{l}\text { Categorías de aspectos } \\
\text { importantes }\end{array}$ & $\begin{array}{l}\text { Categorías de aspectos } \\
\text { prioritarios }\end{array}$ & $\begin{array}{l}\text { Categorías de aspectos } \\
\text { imprescindibles }\end{array}$ \\
\hline Informante 1 & $\begin{array}{l}\text { Psicoanalista } \\
\text { Mediador } \\
\text { Líder } \\
\text { Feliz }\end{array}$ & $\begin{array}{l}\text { Carismático } \\
\text { Experto } \\
\text { Paciente } \\
\text { Amigo } \\
\text { Expresivo } \\
\text { Moderador }\end{array}$ & $\begin{array}{l}\text { Comprensivo } \\
\text { Amable } \\
\text { Cercano } \\
\text { Orientador-guía } \\
\text { Consejero } \\
\text { Buena escucha } \\
\text { Enérgico }\end{array}$ \\
\hline Informante 2 & $\begin{array}{l}\text { Mediador } \\
\text { Paciente } \\
\text { Enérgico }\end{array}$ & $\begin{array}{l}\text { Orientador-guía } \\
\text { Cercano } \\
\text { Consejero } \\
\text { Feliz }\end{array}$ & $\begin{array}{l}\text { Comprensivo } \\
\text { Amable } \\
\text { Experto }\end{array}$ \\
\hline Informante 3 & $\begin{array}{l}\text { Carismático } \\
\text { Líder } \\
\text { Locuaz }\end{array}$ & $\begin{array}{l}\text { Orientador-guía } \\
\text { Psicoanalista } \\
\text { Cercano } \\
\text { Amigo } \\
\text { Feliz } \\
\text { Paciente } \\
\text { Buena escucha } \\
\text { Enérgico }\end{array}$ & $\begin{array}{l}\text { Moderador } \\
\text { Comprensivo } \\
\text { Amable } \\
\text { Experto } \\
\text { Mediador } \\
\text { Consejero }\end{array}$ \\
\hline
\end{tabular}

La convergencia de aspectos imprescindibles, prioritarios o importantes para el ejercicio de una tutoría virtual afectiva no parece estar supeditada a variables relacionadas con la condición auditiva del informante según las informaciones obtenidas en este estudio. Sin embargo, se obtiene una información concluyente y novedosa que resalta la importancia de tener en cuenta aspectos basados en un eLearning afectivo que garanticen la satisfacción del alumnado. En coherencia con lo expuesto en los subapartados anteriores, el tutor, además de experto académico en la temática, ha de cultivar su rol de orientador-guía y consejero desde una metodología docente creativa y variada. Así mismo, ha de incidir de forma obligatoria en los modos en que aborda tanto el trato directo con el alumnado como la planificación de tiempos y las decisiones evaluativas; haciendo que su actuación tutorial se estructure en torno a una actitud comprensiva y amable que le acerque al grupo humano con el que va a compartir la experiencia formativa.

\section{CONCLUSIONES}

De los análisis y los resultados obtenidos se pueden extraer las siguientes conclusiones: 1) Que el modelo de acción tutorial virtual requiere de una serie de ajustes basados en el establecimiento de un eLearning afectivo que incida en una acción tutorial impregnada de afectividad como elemento transversal a todas las funciones y decisiones docentes. 2) Que la tutoría virtual afectiva requiere de una serie de aspectos formativos orientados a la asunción de actitudes positivas basadas en la comprensión, la amabilidad y la empatía que propicien el estado de flujo en el alumnado participante.

\section{REFERENCIAS}

Arranz, V., D. Aguado y B. Lucía, La influencia del tutor en el seguimiento de programas eLearning. Estudio de acciones en un caso práctico, doi: http://dx.doi.org/10.4321/S1576-59622008000100001, Revista de psicología del trabajo y de las organizaciones (en línea), 24(1), 5-23 (2008).

Bautista, G., F. Borges y A. Forés, Didáctica universitaria en Entornos Virtuales de Enseñanza-Aprendizaje, Narcea, Madrid, España (2006). 
Booth, T. y M. Ainscow, Índice de Inclusión. Desarrollando el aprendizaje y la participación en las escuelas, CSIE, Bristol, Reino Unido (2000). http://www.eenet.org.uk/resources/docs/lndex\%20Spanish\%20South\% 20America\%20.pdf Acceso: 28 de mayo de 2014.

Cabero, J. La función tutorial en la teleformación, En Nuevas Tecnologías y Educación por F. Martínez y M.P. Prendes, 15-20, Pearson, Madrid, España (2004).

Cardona, H.E., Consideraciones acerca de la educación virtual como comunidad de relaciones afectivovalorativas, Revista Iberoamericana de Educación, ISSN: 1681-5653 (en línea), 46 (7), 1-10. (2008). http://www.rieoei.org/deloslectores/2203Carmona.pdf Acceso: 28 de mayo de 2014.

Cook, T.D. y CH. S. Reichardt, Métodos cualitativos y cuantitativos en investigación evaluativa, 5. a edición, Morata, Madrid, España (2005).

Csikszentmihalyi, M. El flujo, En Emociones positivas por E. G. Fernández-Abascal (coord.), 181-196, Pirámide, Madrid, España (2009).

Echeita, G., C. Simón, M. López y C. Urbina, Educación inclusiva. Sistemas de referencia, coordinadas y vórtices de un proceso dilemático, En Discapacidad e inclusión. Manual para la docencia por Verdugo, M.A. y R.L. Schalock, 329-358, Amarú, Salamanca, España (2013).

Escalante, J., Enfrentando los nuevos retos educativos. Una mirada profunda a los entornos educativos, mediante un análisis sistemático y consecuente, dando como resultado propuestas fundamentadas en la solución de las problemáticas educativas actuales, Revista Educación hoy, 1-12. (2012).

García Aretio, L. (coord.), De la educación a distancia a la educación virtual, Ariel, Barcelona, España (2007).

Garrison, D.R. y T. Anderson, El E-Learning en el siglo XXI: investigación y práctica, Octaedro, Barcelona, España (2005).

Hernández-Sánchez, A.M. y R. García, ELearning afectivo: indicador clave para el desarrollo de una acción tutorial virtual inclusiva, En Para uma formação virtual acessível e de qualidade por A. Moreira, L. Bengochea y J.R. Hilera, 144-153, Lisboa, Portugal (2013).

http://www.esvial.org/cafvir2013/documentos/LibroActasCAFVIR2013.pdf Acceso: 28 de mayo de 2014.

Klinger, C. L., C. M. Mejía y L. M. Posada, La inclusión educativa: un escenario de expresiones afectivas como mediadoras del aprendizaje, Plumilla Educativa, ISSN-e 1657-4672 (en línea), 8 (12), (2011). http://dialnet.unirioja.es/servlet/articulo?codigo=3801120 Acceso: 28 de mayo de 2014 .

Llorente, M. C., El tutor en e-learning: aspectos a tener en cuenta, Revista Electrónica de Tecnología Educativa, ISSN: 1135-9250 (en línea), 20, 1-24. (2006).

http://edutec.rediris.es/Revelec2/revelec20/llorente.pdf Acceso: 7 de mayo de 2014.

Mallimaci, F. y V. Giménez, Historias de vida y método biográfico, En Estrategias de investigación cualitativa por I. Vasilachis, 175-212, Gedisa, Madrid, España, (2006).

McMillan, J.H. y S. Schumacher, Investigación educativa: una introducción conceptual, Pearson, Madrid, España (2005).

Njenga, J.K. y L.C.H. Fourie, The myths about e-learning in higher education, doi: 10.1111/j.14678535.2008.00910.x, British Journal of Education Technology (en línea), 41 (2), 199-212. (2010).

O'Regan, K., Emotion and eLearning, Journal of Asynchronous Learning Networks (JALN), ISSN: 10928235 (en línea), 7 (3), 78-92. (2003).

http://www.adesignmedia.com/onlineresearch/emotion_learnv7n3_oregan.pdf Acceso: 4 de junio de 2014.

Ortega, J.A., A.M. Hernández-Sánchez, y A. Ortega, Affective eLearning: bienestar en educación a distancia en entornos virtuales. II Congreso Nacional de Psicología Positiva, Castellón, España (2014).

Ozkan, S. y R. Koseler, Multi-dimensional student,s evaluation of e-learning systems in the higher education context: An empirical investigation, doi: 10.1016/j.compedu.2009.06.011, Computers and Education (en línea), 53, 1285-1296. (2009). 
Puello, P., D. Fernández y A. Cabarcas, Herramienta para la detección de estilos de aprendizaje en estudiantes utilizando la plataforma MOODLE, doi: 10.4067/S0718-50062014000400003, Formación Universitaria (en línea), 7 (4), 15-24. (2014).

Rubio, F. y C. García, La experiencia de fluidez en la educación, En Aplicación educativas de la psicología positiva por Caruana, A. et al, 90-109, Generalitat valenciana, Valencia, España (2010).

Seoane, A.M. y F.J. García, Los orígenes del tutor: fundamentos filosóficos y epistemológicos de la monitorización para su aplicación en contextos de e-Learning, En tutoría virtual y e-moderación en red, monográfico en línea, Revista electrónica teoría de la educación. Educación y cultura en la sociedad de la información, ISSN 1138-9737 (en línea), 8, (2), 12-30. (2007).

http://www.usal.es/ teoriaeducacion/rev_numero_08_02/n8_02_seoane_garcia.pdf Acceso: 7 de mayo de 2014.

Shen, L., M. Wang y R. Shen, Affective e-Learning: using "emotional" data to improve learning in pervasive learning environment, Educational Technology and Society, ISSN 1436-4522 (en línea), 12 (2), 176-189. (2009). http://www.ifets.info/journals/12_2/13.pdf Acceso: 4 de junio de 2014.

Stake, R. E., Evaluación comprensiva y evaluación basada en estándares, Graó, Barcelona, España (2006).

Suárez, J.M. y D. Anaya, Educación a distancia y presencial: diferencias en los componentes cognitivo y motivacional de estudiantes universitarios, Revista Iberoamericana de Educación a Distancia, RIED, I.S.S.N.:1138-2783 (en línea), 7, 65-75. (2004). http://www.utpl.edu.ec/ried/images/pdfs/vol7-12/educacion_distancia_presencial.pdf Acceso: 7 de mayo de 2014.

Tarragona, M. Psicología positiva y terapias constructivistas: una propuesta integradora, doi: http://dx.doi.org/10.4067/S0718-48082013000100011, Terapia psicológica (en línea), 31 (1), 115-125. (2013).

Vázquez, C., La ciencia del bienestar psicológico, En La ciencia del bienestar. Fundamentos de una psicología positiva por Vázquez, C. y G. Hervás, 13-46, Alianza, Madrid, España (2009).

Zaharias, P. y A. Poylymenakou, Developing a usability evaluation method for e-learning applications: beyond funtional usability, doi: 10.1080/10447310802546716, International Journal of Human-Computer Interaction (en línea), 25 (1), 75-98. (2009). 